\title{
A simple and effective process for noise reduction of multichannel cortical field potential recordings in freely moving rats
}

\author{
Fu-Zen Shaw ${ }^{\mathrm{a}, *}$, Chen-Tung Yen ${ }^{\mathrm{b}}$, Ruei-Feng Chen ${ }^{\mathrm{b}}$ \\ ${ }^{a}$ Institute of Neuroscience, Tzu Chi University, No. 701, Chung Yang Rd. Sec. 3, Hualien 970, Taiwan \\ ${ }^{\mathrm{b}}$ Department of Zoology, National Taiwan University, Taipei, Taiwan
}

Received 13 September 2002; received in revised form 7 December 2002; accepted 10 January 2003

\begin{abstract}
Simple and useful steps, i.e. placing a grounded plate under the recording chamber as well as using multiple reference electrodes, are introduced here for obtaining reliable low-noise recordings of brain activity in freely moving rats. A general circuit model was built to analyze the electrical interference of both single-grounded and two-reference ground-free recording configurations. In both simulated and realistic conditions under two recording states, $60-\mathrm{Hz}$ magnitude was in the microvolt range. Moreover, the noise was significantly reduced by shortening the distance between the subject and the grounded plate under the recording chamber. Furthermore, in chronically implanted rats, average $60-\mathrm{Hz}$ interference of multichannel electroencephalograms of two-reference ground-free recordings $(3.74 \pm 0.18 \mu \mathrm{V})$ was significantly smaller than that of the single-grounded condition $(9.03 \pm 1.98 \mu \mathrm{V})$. Thus, we demonstrated that a lower-noise recording can be achieved by a two-reference configuration and a closely-placed metal grounded plate in an open-field circumstance. As compared to the use of a Faraday cage, this simple procedure is of benefit for long-term behavioral tracking with a video camera and for pharmacological experiments.
\end{abstract}

(C) 2003 Elsevier Science B.V. All rights reserved.

Keywords: Multichannel recording; Conscious animal; Long-term recording; EEG; Evoked potential; Faraday cage

\section{Introduction}

Cortical field potentials, which reflect summated activities of numerous cortical neurons, reveal distinct patterns in different natural states, for example, gamma activity, sleep spindle, and theta wave (Steriade, 2000). These brain waves are often used to characterize vigilance states or indicate different information processes (Maloney et al., 1997; Buzsáki, 1998; Steriade, 2000). To specify these brain activities and figure out their spatial/temporal relationships among different brain areas, a low-noise multichannel recording in conscious subjects is very important. A prominent interference is primarily caused by the power line nearby the subject. Several techniques have been proposed to reduce the interference in human electrophysiological studies. Twisting the recording wire (Huhta and Web-

\footnotetext{
* Corresponding author. Tel.: +886-3-8565301x7180; fax: +886-38461733.

E-mail address: fzshaw@mail.tcu.edu.tw (F.-Z. Shaw).
}

ster, 1973) or shielding the recording wire (Ott, 1976; Metting van Rijn et al., 1990) can decrease the influence of electromagnetic induction. A higher common mode rejection ratio (CMRR) of the amplifier or other techniques are also helpful for decreasing the electrical interference (Thakor and Webster, 1980; Winter and Webster, 1983; Buzsáki et al., 1989; Metting van Rijn et al., 1990). Theoretically, all recording processes used with human recording should be applicable to animal studies. However, few studies have discussed the utility of those processes in animal recording. For example, an active guarding technique can be used for noise reduction and preservation of the signal frequency (Metting van Rijn et al., 1990), but that also increases the circuitry complexity and the load to the animal. A grounded Faraday cage is a popular apparatus to obtain low-noise recordings in conscious animals (Vergnes et al., 1987; Maloney et al., 1997), but it presents obstacles to video tracking of animal behavior. Does a simple process exist for acquiring low-noise biopotentials in freely moving animals? In the present study, we intro- 
duce a multiple-reference ground-free recording technique in company with a grounded plate, and use a commercial instrumentation amplifier with a 3-operational amplifier (3-OPAMP) configuration to obtain low-noise, low-interference recordings. The experiment was performed in freely moving rats, a common animal model. Results from both simulation and animal experiments indicate that a high-quality recording can be obtained under the two-reference ground-free condition.

\section{Simulation with a model circuitry}

Fig. 1 depicts a model that includes an amplifier and recording electrodes. $Z_{\mathrm{e}}$ and $Z_{\text {ref }}$ are the impedances of the recording and reference electrodes, respectively. $Z_{\mathrm{g}}$ is the ground electrode impedance; $Z_{\mathrm{cm}}$ is the commonmode input impedance of the amplifier. $\mathrm{C}$ represents the capacitive coupling from the power line and other environmental sources to the animal. $C_{1}$ is a capacitor between the subject and the ground. $X_{\mathrm{C}}$ and $X_{C_{1}}$ are the reactive impedances of $C$ and $C_{1}$, respectively. Switch SW determines two recording situations: at the hori-

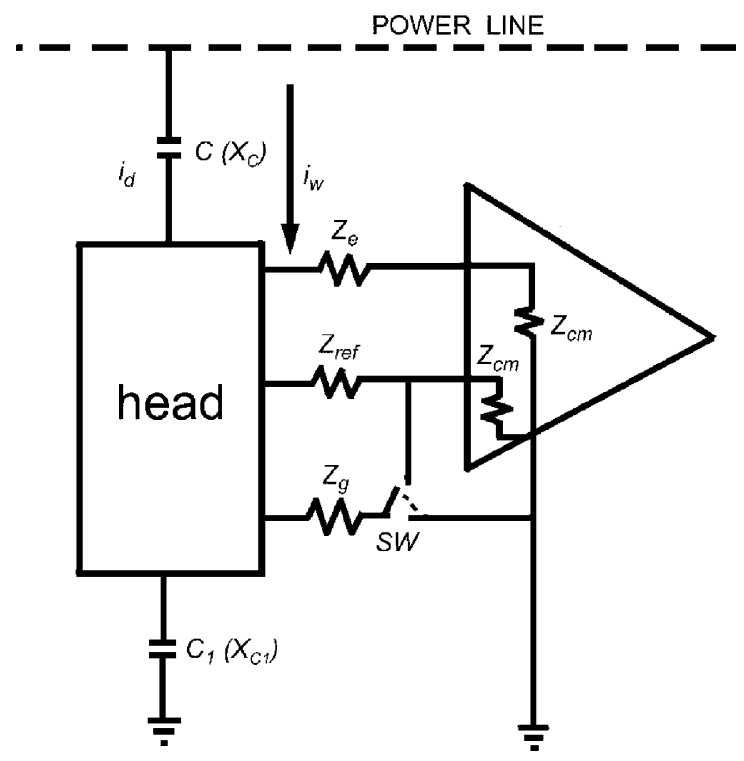

Fig. 1. A circuit model for EEG recording. The large triangle at the right is an operational amplifier. $Z_{\mathrm{e}}$ and $Z_{\mathrm{ref}}$ are the impedances of the recording and reference electrodes, respectively. $Z_{\mathrm{g}}$ is the ground electrode impedance; $Z_{\mathrm{cm}}$ is the common-mode input impedance of the amplifier. $C$ represents the capacitive coupling from the power line and other environmental sources to the animal. $C_{1}$ is a capacitor between the subject and the ground. $X_{C}$ and $X_{C_{1}}$ are the reactive impedances of $C$ and $C_{1}$, respectively. Two prominent displacement currents from the powerline are considered here: first, the displacement current, $i_{\mathrm{w}}$, flowing through two EEG leads; second, the displacement current, $i_{\mathrm{d}}$, flowing through the ground electrode (grounded recording) or capacitor $C_{1}$ (ground free recording). Switching SW to the horizontal position creates a grounded configuration. When it is switched upwards, the circuitry becomes a ground-free configuration with two reference electrodes. zontal position, the grounded recording; and flipped upwards, the ground-free recording with two reference electrodes.

Two major sources of electrical interference in biopotential recordings have been described: electromagnetic induction and electrostatic induction. Several authors have analyzed the contribution of each source and ways to reduce the interference (Huhta and Webster, 1973; Ott, 1976; Thakor and Webster, 1980; Metting van Rijn et al., 1990). For example, magnetically induced interference is proportional to the flux density and the surface area enclosed by the leads within the magnetic field. This can be reduced by twisting the leads together. Keeping the amplifier away from any AC power sources is also useful for reducing electromagnetic interference.

As compared to the contribution of electromagnetic induction, another prominent noise source is generated by electrical displacement currents through various compartments. The interference induced by displacement currents through body tissue is very small because electroencephalographic (EEG) electrodes are often placed close together (i.e. with low impedance between electrodes), therefore its contribution can be ignored (Huhta and Webster, 1973). By contrast, the two most important types of interferences are the voltage drops induced by both the displacement current, $i_{\mathrm{w}}$, flowing through the EEG leads and the common-mode voltage generated by the displacement current, $i_{\mathrm{d}}$, flowing through the ground electrode (Fig. 1). We focus on these two contributions. With grounded recordings, the ground electrode, $Z_{\mathrm{g}}$, is switched to connect with the ground (Fig. 1). Therefore the electrical interference, $V_{\mathrm{n}}$, can be presented by the following equation (Huhta and Webster, 1973; Thakor and Webster, 1980):

$$
\begin{aligned}
V_{\mathrm{n}}= & i_{\mathrm{w}}\left(Z_{\mathrm{e}}-Z_{\mathrm{ref}}\right) \\
& +i_{\mathrm{d}} Z_{\mathrm{g}}\left[\frac{1}{\mathrm{CMRR}}+\left(\frac{Z_{\mathrm{cm}}}{Z_{\mathrm{cm}}+Z_{\mathrm{e}}}-\frac{Z_{\mathrm{cm}}}{Z_{\mathrm{cm}}+Z_{\mathrm{ref}}}\right)\right] .
\end{aligned}
$$

Since usually $Z_{\mathrm{cm}} \gg Z_{\mathrm{e}}, Z_{\text {ref }}$ and $Z_{\mathrm{d}}=\left|Z_{\mathrm{e}}-Z_{\text {ref }}\right|$,

$$
V_{\mathrm{n}}=i_{\mathrm{w}} Z_{\mathrm{d}}+i_{\mathrm{d}} Z_{\mathrm{g}}\left[\frac{1}{\mathrm{CMRR}}+\frac{Z_{\mathrm{d}}}{Z_{\mathrm{cm}}}\right] \text {. }
$$

The interference caused by common-mode signals can be reduced by a higher CMRR of the differential amplifier and a higher common-mode input impedance $\left(Z_{\mathrm{cm}}\right)$ of the amplifier. With grounded recordings, the interference is also dependent on the impedance difference between the recording electrodes, $Z_{\mathrm{d}}$. Thus a decreased $Z_{\mathrm{d}}$ will result in lower electrical interference. Generally, the difference in impedances of the two recording electrodes is $10 \mathrm{k} \Omega$, and the CMRR and $Z_{\mathrm{cm}}$ of an instrumentation preamplifier will be $110 \mathrm{~dB}$ and 10 $\mathrm{G} \Omega$, respectively (see Section 3). The displacement current, $i_{\mathrm{d}}$, is typically $0.1 \mu \mathrm{A}$ (Huhta and Webster, 
1973; Thakor and Webster, 1980). The displacement current, $i_{\mathrm{w}}$, is dependent on the architecture of the recording wire and is typically $1 \mathrm{nA}$ (Huhta and Webster, 1973; Wood et al., 1995). The contributions of $i_{\mathrm{w}}$ and $i_{\mathrm{d}}$ to the interference are 10 and $0.011 \mu \mathrm{V}$, respectively. Thus the total electrical interference of grounded recordings is about $10 \mu \mathrm{V}$. In this case, the interference is strongly dependent on the displacement current, $i_{\mathrm{w}}$, through the EEG lead.

Under a ground-free recording configuration, the animal is no longer grounded. The ground lead is connected to the reference lead to constitute two references in the rat (Fig. 1). The displacement current, $i_{\mathrm{d}}$, now flows through the high-impedance paths of $X_{C_{1}}$ and $Z_{\mathrm{cm}}$. The common-mode voltage is high too. The electrical interference is given by

$$
\begin{aligned}
V_{\mathrm{n}}= & i_{\mathrm{w}}\left(Z_{\mathrm{e}}-Z_{\mathrm{p}}\right)+\frac{i_{\mathrm{d}} Z_{\mathrm{cm}}}{2} \frac{X_{C_{1}}}{\sqrt{X_{C_{1}}^{2}+Z_{\mathrm{cm}}^{2} / 4}} \\
& \times\left[\frac{1}{\mathrm{CMRR}}+\left(\frac{Z_{\mathrm{cm}}}{Z_{\mathrm{cm}}+Z_{\mathrm{e}}}-\frac{Z_{\mathrm{cm}}}{Z_{\mathrm{cm}}+Z_{\mathrm{p}}}\right)\right],
\end{aligned}
$$

where $Z_{\mathrm{p}}=Z_{\mathrm{g}} Z_{\text {ref }} / Z_{\mathrm{g}}+Z_{\text {ref }}$

We can simplify the equation as

$$
\begin{aligned}
V_{\mathrm{n}}= & \frac{1}{2} i_{\mathrm{w}}\left(Z_{\mathrm{e}}-Z_{\mathrm{p}}\right)+i_{\mathrm{d}} \frac{X_{C_{1}}}{\sqrt{4 X_{C_{1}}^{2} / Z_{\mathrm{cm}}^{2}+1}} \\
& \times\left[\frac{1}{\mathrm{CMRR}}+\frac{Z_{\mathrm{e}}-Z_{\mathrm{p}}}{Z_{\mathrm{cm}}}\right] .
\end{aligned}
$$

Generally, $Z_{\mathrm{cm}} \gg X_{C_{1}}$, and thus

$V_{\mathrm{n}} \cong i_{\mathrm{w}}\left(Z_{\mathrm{e}}-Z_{\mathrm{p}}\right)+i_{\mathrm{d}} X_{C_{1}}\left[\frac{1}{\mathrm{CMRR}}+\frac{Z_{\mathrm{e}}-Z_{\mathrm{p}}}{Z_{\mathrm{cm}}}\right]$.

In an ideal condition, $Z_{\mathrm{p}}$ will approximate $Z_{\mathrm{ref}} / 2$, and thus Eq. (2) can be written as

$V_{\mathrm{n}} \cong \frac{1}{2} i_{\mathrm{w}} Z_{\mathrm{d}}+i_{\mathrm{d}} X_{C_{1}}\left[\frac{1}{\mathrm{CMRR}}+\frac{Z_{\mathrm{e}}+Z_{\mathrm{d}}}{2 Z_{\mathrm{cm}}}\right]$.

The electrical interference can be reduced by a higher CMRR and $Z_{\mathrm{cm}}$ of the amplifier. This is similar to the grounded recording configuration. However, the interference of ground-free recordings is dependent on $X_{C_{1}}$, not on the impedance of the ground electrode. That is, decreasing $X_{C_{1}}$ is helpful in reducing the electrical interference in the ground-free recording configuration. A typical value of $X_{C_{1}}$ is $\sim 10 \mathrm{M} \Omega$ (Huhta and Webster, 1973; Thakor and Webster, 1980). Therefore, the contributions of $i_{\mathrm{w}}$ and $i_{\mathrm{d}}$ to the interference are 5 and 11 $\mu \mathrm{V}$, respectively. Thus total electrical interference of ground-free recordings is $16 \mu \mathrm{V}$. In this case, the interference is relatively dependent on the displacement current, $i_{\mathrm{d}}$. Furthermore, shortening the distance between the animal and the ground can significantly decrease both the $X_{C_{1}}$ value and the inductive current from the power line, thus resulting in a decrease in the interference to a value far less than $15 \mu \mathrm{V}$ (see Section 4).

\section{Experimental setup}

Male Wistar rats were anesthetized with sodium pentobarbital $(50 \mathrm{mg} / \mathrm{kg}$, i.p., $n=10)$. After being anesthetized, the rat was placed in a stereotaxic apparatus, the dorsal surface of the rat's head was cleaned, and stainless steel screw electrodes were driven into the skull. Care was taken not to damage the dura. Twelve recording electrodes were implanted in various cortical areas. The detailed location of the electrodes on the skull has been depicted in a previous study (Shaw et al., 1999a). A reference and a ground electrode were placed in the skull over the cerebellum. The skull over the cerebellum is usually thicker than over the cerebral area. Dental cement was applied to the skull screws, the skull surface, and the connector to secure them in place. The average impedance of 30 randomly selected screw electrodes was about $3 \mathrm{k} \Omega(1.5-10 \mathrm{k} \Omega)$, which was measured from a $100-\mathrm{Hz}, 10-\mathrm{mV}$ sinusoidal wave passing through a serially connected $1-\mathrm{M} \Omega$ resistor. Rats were housed individually in cages and injected with antibiotics after surgery. Three weeks following recovery from surgery, a rat was placed in a transparent acrylic chamber five times $(2 \mathrm{~h} /$ day $)$ to habituate it to the experimental environment. On the day of the experiment, the animal was placed in the recording chamber for $30 \mathrm{~min}$ prior to the recording session. The experiment was performed in a well-controlled and soundattenuated room. After completion of the experiment, the animal was sacrificed with an overdose of sodium pentobarbital. Special attention was given to ascertain whether the screw electrodes had penetrated through the skull but had not penetrated the dura. All surgical and experimental procedures were approved by the Experimental Animal Care and Use Committee of Tzu Chi University.

The entire recording and monitoring system includes an isolated AC power unit, an oscilloscope, a multichannel amplifier for EEG activity, and a general data acquisition system (Fig. 2). The independent AC power unit was helpful in isolating external interference from the electric power system of the building. A customfabricated shielded multistrand cable line was used to conduct the cortical electrical activity into a multichannel amplifier. To reduce distortion of the brain activity and noise contamination, a commercial 3OPAMP instrumentation amplifier (AD620, Analog Devices, PMI Division) was selected as the preamplifier here because of its high input impedance $(10 \mathrm{G} \Omega)$ and good CMRR (110 dB with a gain of 10). The preamplified signal was then amplified again and filtered in 


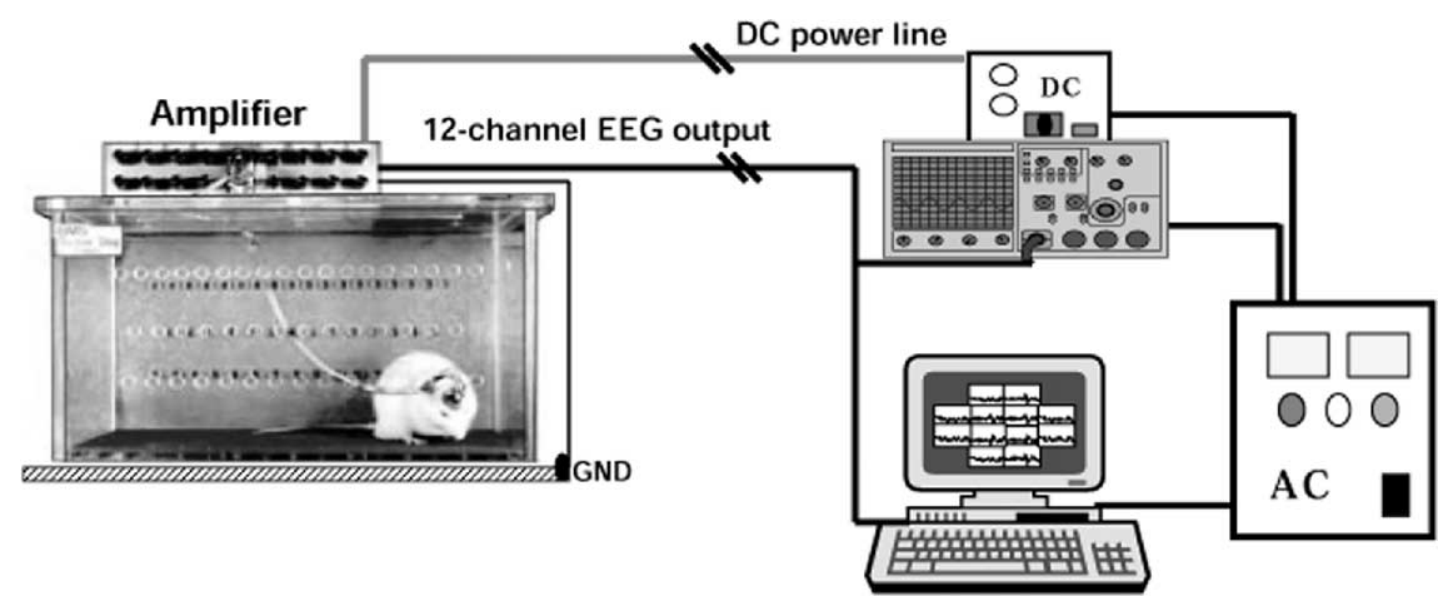

Fig. 2. System setup for multichannel recordings in freely moving rats. The entire system includes an AC power unit, an oscilloscope, a multichannel amplifier, and a data acquisition system. In order to reduce power line-related interference, all power sources and the data acquisition system were kept 1-2 $\mathrm{m}$ away from the recording box and amplifier. The independent AC power unit was helpful in isolating external interference from the electric power system of the building. A metal plate with a single grounded end was placed under the recording box with the distance of 5 mm. GND, ground; DC, DC power supply; AC, AC power unit.

the range of $0.3-1000 \mathrm{~Hz}$ (4th order). The detailed circuitry of the multichannel amplifier was described in a previous study (Shaw et al., 1999a). A DC power supply, which was made by two general voltage regulators with larger capacitors, was kept 1-2 m away from the multichannel amplifier. This was helpful in reducing the noise from the $\mathrm{AC}$ power line and the transformer. In addition, a metal plate with a singlepoint ground was placed under the recording box. In our preparation, the distance between the recording box and the metal plate was $5 \mathrm{~mm}$. This arrangement increased the capacitance between the animal and ground, thus leading to reduced interference of the recorded biopotentials (see Section 4).

In a general design for electrophysiological recordings, both reference lead and a ground lead are necessary. The reference and ground electrodes were connected to the negative input of the preamplifier and the amplifier's ground, respectively. This constituted the grounded recording configuration. By contrast, the ground-free recording was made by directly connecting both the ground and the reference wires to the negative input of the instrumentation amplifier. If the ground wire is disconnected, the system is also a ground-free recording. However, the open wire will act as an antenna then pick up more external noise. It will create more difficulty for the evaluation of noise contribution. Moreover, the same recording multiwire used in both recording modes will reduce the complexity of the evaluation of noise contribution. Therefore, the ground-free recording with two reference electrodes was used here. The recorded brain activity was transferred to the frequency domain using Fourier transformation with a Hamming window. Data acquisition and the analytic programs were described elsewhere in detail (Shaw et al., 1999a).

\section{Results}

To evaluate the contribution to noise of the distance between the animal and the grounded plate, an artificial rat was made from a rat doll covered with metal foil. The recording, reference, and ground electrodes were replaced with precise resistors of 1,10 , and $10 \mathrm{k} \Omega(1 \%$ error), respectively. The noise level, calculated from 20 randomly selected 2-s data segments, significantly decreased with shorter distances between the artificial rat and the grounded plate under both recording modes (Fig. 3; linear regression analysis, $P<0.0001$ ). The noise reduction might be caused by lower current induction from the power line because there were no compartment changes during the recording session under grounded recording. In addition, the capacitance, measured with a general capacitance meter, increased as the distance between the artificial rat and the grounded plate was shortened (Table 1). Except for the lower displacement current, a higher capacitance will also result in reducing the contribution of $60-\mathrm{Hz}$ interference (Fig. 2). Apparently, the noise of the two-reference ground-free recording was lower than that of the single-grounded recording with a shorter distance between the rat and grounded plate (Table 1). The resistance imbalance $(4 \mathrm{k} \Omega)$ in ground-free recording is smaller than that $(9 \mathrm{k} \Omega)$ of grounded recording. According to Eq. (2), the noise of ground-free recording will be lower than that of grounded recording. Therefore, combining two reference electrodes was helpful in reducing the noise contamination.

Single-channel cortical activity of a chronically implanted rat is illustrated in Fig. 4. No apparent $60-\mathrm{Hz}$ noise could be observed in temporal traces under either recording mode. However, clear $60-\mathrm{Hz}$ interference was detected in the power spectra of grounded recordings 

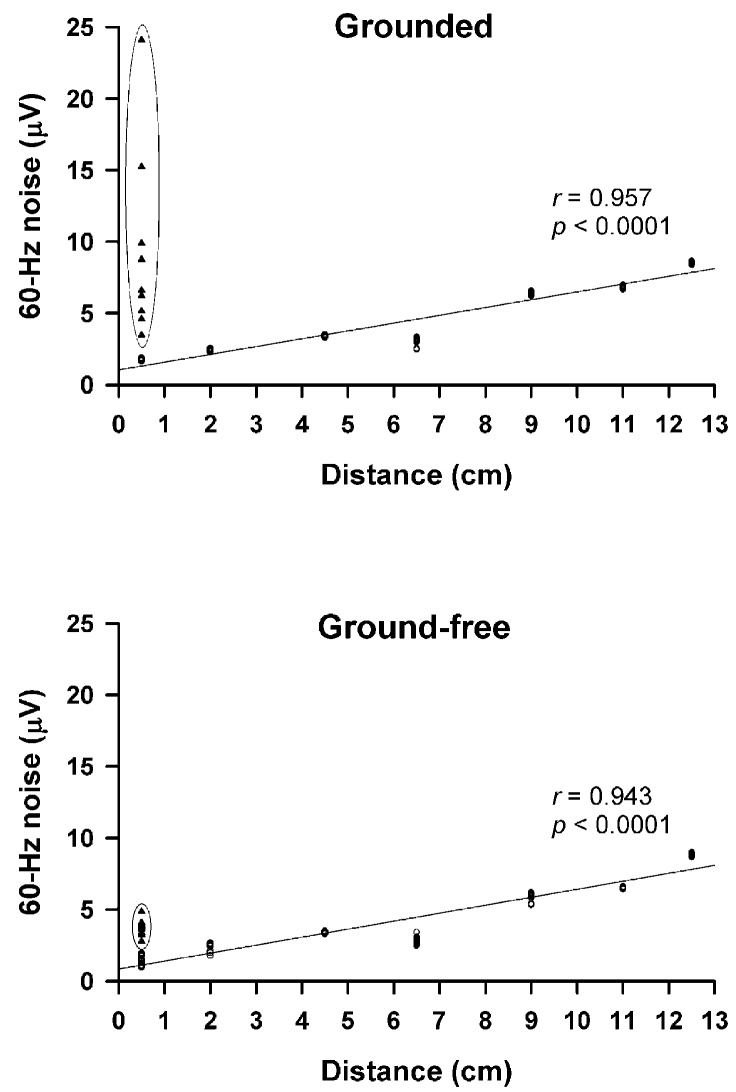

Fig. 3. Distribution of $60-\mathrm{Hz}$ magnitudes of grounded and groundfree recordings using different distances between the artificial rat and the underlying grounded plate. Twenty $60-\mathrm{Hz}$ magnitudes (open circles) were measured under an identical distance. The $60-\mathrm{Hz}$ magnitude decreased as the distance was shortened for both recordings with a significant linear relationship. The $60-\mathrm{Hz}$ noise values of chronically implanted rats under both recordings (filled triangles) are circled. The distribution of $60-\mathrm{Hz}$ magnitude of ground-free recording is more convergent than that of grounded recording.

Table 1

Averaged $60-\mathrm{Hz}$ magnitudes of single-grounded and two-reference ground-free recordings and capacitance with different distances between the artificial rat and underlying grounded plate

\begin{tabular}{llrl}
\hline Distance $(\mathrm{cm})$ & Grounded $(\mu \mathrm{V})$ & Ground-free $(\mu \mathrm{V})$ & Capacitance $(\mathrm{pF})$ \\
\hline 12.5 & $8.52 \pm 0.02^{\mathrm{a}}$ & $8.88 \pm 0.02$ & 7.6 \\
11 & $6.79 \pm 0.02$ & $6.5 \pm 0.01$ & 11.7 \\
9 & $6.37 \pm 0.02$ & $5.96 \pm 0.06$ & 12 \\
6.5 & $3.07 \pm 0.05$ & $2.81 \pm 0.06$ & 13.4 \\
4.5 & $3.41 \pm 0.02$ & $3.39 \pm 0.01$ & 13.8 \\
2 & $2.41 \pm 0.03$ & $2.4 \pm 0.05$ & 15.5 \\
0.5 & $1.78 \pm 0.04$ & $1.53 \pm 0.06$ & 30.5 \\
\hline
\end{tabular}

\footnotetext{
${ }^{\text {a }}$ Values were calculated from 20 randomly selected 2-s data segment. Data are the mean \pm standard error.
}

(arrow in Fig. 4), but no obvious $60-\mathrm{Hz}$ peak occurred with ground-free recordings. The average interference calculated from 12 cortical sites in each individual rat is summarized in Table 2. Average $60-\mathrm{Hz}$ interferences readings of the grounded and ground-free recordings were $9.03 \pm 1.98$ and $3.74 \pm 0.18 \mu \mathrm{V}$, respectively. The electrical interference in the ground-free condition was significantly less variant than that in the grounded condition (circles in Fig. 3; repeated-measures ANOVA, $P<0.02$ ). In addition, the noise contamination of the ground-free recording was a half or less of that of the grounded recording (Student-Newman-Keuls method, $P<0.05)$.

At the end of the experiment, we found that the reference electrodes had not completely penetrated the bone over the cerebellum in two rats (rats 7 and 8 in Table 2). In an additional acute experiment, we found the impedances of non-penetrating screws at $60 \mathrm{~Hz}$ attained $85 \mathrm{k} \Omega(18-85 \mathrm{k} \Omega)$, whereas those of penetrating screws were close to $7.5 \mathrm{k} \Omega(5-12 \mathrm{k} \Omega)$.

\section{Discussion}

A typical EEG has an amplitude of approximately $100 \mu \mathrm{V}$. Therefore, a tolerable electrical interference, $V_{\mathrm{n}}$, should be smaller than $10 \mu \mathrm{V}$, i.e. signal-to-noise ratio $>10$. This objective was achieved in both the grounded and ground-free recordings (Table 2). In other words, the procedures described in the experimental setup section, e.g. shielding of the recording leads, isolation of the AC power unit, separation of the DC power supply, use of an instrumentation amplifier with a higher CMRR and higher $Z_{\mathrm{cm}}$, etc. has effectively reduced external interferences (Huhta and Webster, 1973; Ott, 1976; Thakor and Webster, 1980; Winter and Webster, 1983; Metting van Rijn et al., 1990). Two reference electrodes in the ground-free recordings also led to noise reduction (Eq. (2), Tables 1 and 2). Moreover, shortening the distance between the subject and the grounded plate decreased the noise contribution (Fig. 3). The addition of a grounded plate is also useful for noise reduction in other recording systems, such as with a Grass amplifier (model 7P511). In addition, a grounded plate for the noise reduction has also been demonstrated in the single-ended recording (Shaw et al., 2002). With all these precautions taken, we found that very low $60-\mathrm{Hz}$ interference was obtained in the tworeference ground-free recording with a grounded plate (Table 2).

The noise level of ground-free recordings was more stable than that of grounded recordings (Fig. 3, Table 2 ). Why do ground-free recordings have a better and more stable performance? The noise is attributed to different factors in the two types of recordings. For grounded recordings, the interference is dependent on the difference in impedances of the two recording electrodes, $Z_{\mathrm{d}}$ (Eq. (1)). In the two worst cases (rats 7 and 8 in Table 2), the reference electrode did not penetrate all the way through the skull. In an acute experiment, we found that the impedance of non- 

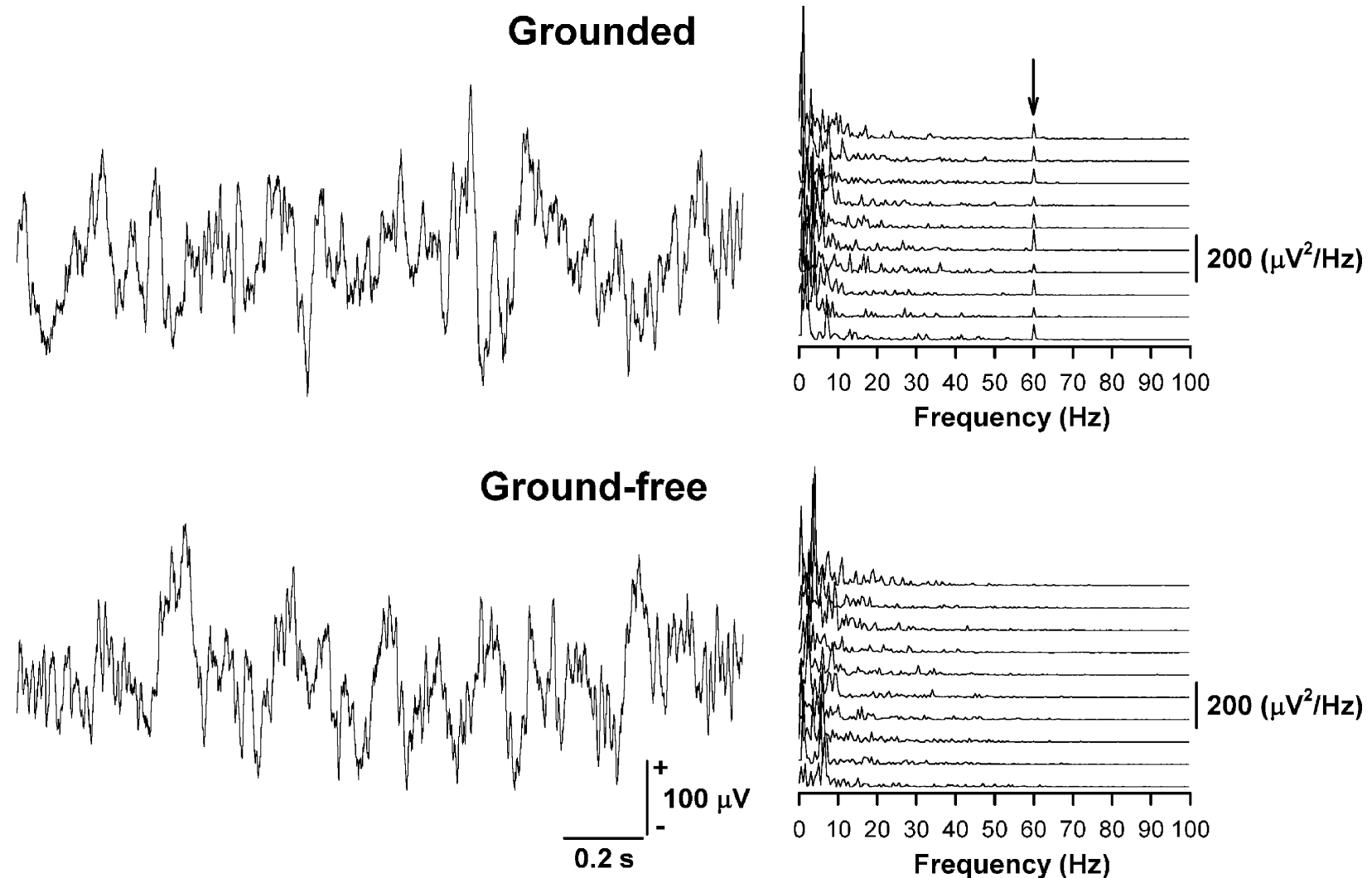

Fig. 4. An example of temporal (left panel) and spectral (right panel) activities of a single-channel EEG under grounded and ground-free recordings. No obvious $60-\mathrm{Hz}$ interference was observed by visual inspection of the temporal EEG for either recording, but clear 60-Hz peaks (arrow) were depicted in 10 consecutive EEG spectra for the grounded recordings. Mean magnitudes of the 12-channel EEGs under grounded and ground-free recordings at $60 \mathrm{~Hz}$ were 24.1 and $4.85 \mu \mathrm{V}$, respectively.

Table 2

Comparison of averaged EEG magnitudes $(\mu \mathrm{V})$ at $60 \mathrm{~Hz}$ under single grounded and two-reference ground-free recordings

\begin{tabular}{lll}
\hline Rat number & Grounded & Ground-free \\
\hline 1 & 9.89 & 3.37 \\
2 & 5.15 & 3.82 \\
3 & 6.6 & 3.63 \\
4 & 4.6 & 4.08 \\
5 & 6.21 & 3.72 \\
6 & 8.76 & 2.78 \\
7 & 15.24 & 3.95 \\
8 & 24.1 & 4.85 \\
9 & 6.22 & 3.94 \\
10 & 3.48 & 3.22 \\
Average & $9.03 \pm 1.98$ & $3.74 \pm 0.18^{*}$ \\
\hline
\end{tabular}

Data are the mean \pm standard error.

* $P<0.05$, by the Student-Newman-Keuls method.

penetrated reference electrode could attain $85 \mathrm{k} \Omega(18-$ $85 \mathrm{k} \Omega$ ). Therefore, $Z_{\mathrm{d}}$ was in the range of $18-85 \mathrm{k} \Omega$. By contrast, the interference is dependent on $Z_{\mathrm{e}}-Z_{\mathrm{p}}$ for ground-free recordings (Eq. (2)). When $Z_{\text {ref }}$ can attain $85 \mathrm{k} \Omega$ and $Z_{\mathrm{g}}$ is in the range of $5-12 \mathrm{k} \Omega, Z_{\mathrm{p}}$ will be $5-12$ $\mathrm{k} \Omega$. Therefore, $Z_{\mathrm{e}}-Z_{\mathrm{p}}$ will be in the range of 5-12 $\mathrm{k} \Omega$. The difference of attributed factors between single- grounded and two-reference ground-free recordings could be several folds, and the noise discrepancy also fell in the same range (Table 2). Therefore, changes in impedance may account for variations in the electrical interference for grounded recordings. Accordingly, two reference electrodes are useful for decreasing the variation in the attributed factors, then resulting in noise reduction. Although we only used two references with ground-free recordings in the present study, we believe that a similar result would also be achieved for tworeference grounded recordings because the noise level for both recording modes under simulation were identical (Table 1). Based on these results, measuring and reducing the impedance of each skull electrode during surgical procedures plays an important role in acquiring low-noise electrical activity.

In the present study, a contrary result seems to be obtained between theoretical calculation and simulated/ experimental measurements at a glance. In the theoretical calculation, we considered all condition and displacement currents as previous studies used (Huhta and Webster, 1973; Thakor and Webster, 1980; Wood et al., 1995). Previous studies have demonstrated that the noise of ground-free recording is higher than that of grounded recording (Thakor and Webster, 1980; Wood 
et al., 1995). We also got the same results in the theoretical calculation. However, we found that the $60-\mathrm{Hz}$ noise was reduced as distance between the rat and the grounded metal plate was shortened (Fig. 3). Because there was no alteration of hardware under grounded recording mode, the decreased $60-\mathrm{Hz}$ may be due to a decrease of the displacement current. In ground-free recording, a similar trend of the change of $60-\mathrm{Hz}$ magnitude was found (Fig. 3). Changes of the displacement current would be very similar to that of grounded recording. A shortened distance between the rat and grounded plate will also result in decreasing the reactive impedance of $C_{1}$ (Table 1 ). In addition, we selected an instrumentation amplifier with high $Z_{\mathrm{cm}}$ and CMRR as a preamplifier. Based on these parameters, the value in the second term of Eq. (2) will be very close to that of Eq. (1). Accordingly, the impedance imbalance (the first term of Eqs. (1) and (2)) will become a dominant factor in both recording configurations under our preparation. The impedance imbalance in tworeference ground-free recording is smaller and more convergent than that of grounded recording. This is why a higher noise level and wider variance were found under the grounded recording (Table 2). It may also be the reason why a contrary result was found between theoretical calculation and simulated/experimental measurements in the present study.

A grounded metal plate was placed under the recording box during the experiment to decrease $X_{C_{1}}$ as well as the inductive current from the power line (Table 1). A stable and low-noise brain activity recording was obtained (Fig. 3). The phenomenon of reducing the interference can be predicted by Eq. (2). Obviously, placing a grounded plate under the recording chamber and shortening their interdistance is a very simple and useful process to consistently acquire low-noise brain activity. However, shortening the distance between the animal and the grounded plate constitutes a larger capacitor, which can store a greater charge. While temporarily touching the subjects by experimenter will cause the redistribution of the stored charges, then will induce voltage drift of the recording (Thakor and Webster, 1980). However, in most studies brief contact of animals during recording sessions can be avoided. Therefore, the influence of electrostatic currents can be ignored. Under the same preparation, a long-term recording with low-noise contamination was demonstrated in our previous studies (Shaw et al., 1999b, 2002). Based on both simulation and experimental results, the two-reference ground-free technique is a simpler and more robust method for acquiring low-noise biopotentials.

A very popular treatment for noise reduction for electrophysiological experiments is comprised of a recording chamber with a grounded metal cage, i.e. a Faraday cage (Vergnes et al., 1987; Maloney et al.,
1997). However, a Faraday cage is not convenient for video tracking of animal behavior or for drug administration experiments. A simple grounded metal plate described in the present study not only has the advantage of obtaining low-noise electric activity, but also provides greater working spaces to carry out other simultaneous manipulations. This merit was demonstrated in our recent report about long-term, low-noise biopotential recording during sleep-wake states (Shaw et al., 2002). In addition, although a metal plate was used in the present study, a sheet of foil will also lead to similar results.

In summary, we provide a theoretical basis and experimental results to demonstrate that a two-reference recording setup with a grounded metal plate is able to acquire reliable low-noise biopotentials. This technique is not only useful for multichannel recording of brain activities of conscious rats, but also of benefit to longterm behavior tracking and pharmacological testing in freely moving animals.

\section{Acknowledgements}

The authors thank Dr John G. Harris for his thoughtful comments on the manuscript. This work was supported by grants NSC90-2314-B320-010 and NSC91-2745-P320-001 from the National Science Council, Taiwan.

\section{References}

Buzsáki G. Memory consolidation during sleep: a neurophysiological perspective. J Sleep Res 1998;7:17-23.

Buzsáki G, Bickford RG, Ryan LJ, Young S, Prohaska O, Mandel RJ, Gage FH. Multisite recording of brain field potentials and unit activity in freely moving rats. J Neurosci Methods 1989;28:209-17.

Huhta JC, Webster JG. 60-Hz interference in electrocardiography. IEEE Trans Biomed Eng 1973;20:91-101.

Maloney KJ, Cape EG, Gotman J, Jones BE. High-frequency $\gamma$ electroencephalogram activity in association with sleep-wake states and spontaneous behaviors in the rat. Neuroscience 1997;76:54155.

Metting van Rijn AC, Peper A, Grimbergen CA. High-quality recording of bioelectric events. Part 1. Interference reduction, theory and practice. Med Biol Eng Comput 1990;28:389-97.

Ott HW. Noise reduction techniques in electronic systems. New York: John Wiley \& Sons, 1976:54-90.

Shaw F-Z, Lai CJ, Chiu TH. A low-noise flexible integrated system for recording and analysis of multiple electrical signals during sleepwake states in rats. J Neurosci Methods 2002;118:77-87.

Shaw F-Z, Chen R-F, Tsao H-W, Yen C-T. A multichannel system for recording and analysis of cortical field potentials in freely moving rats. J Neurosci Methods 1999a;88:33-43.

Shaw F-Z, Chen R-F, Tsao H-W, Yen C-T. Algorithmic complexity as an index of cortical function in awake and pentobarbital-anesthetized rats. J Neurosci Methods 1999b;93:101-10.

Steriade M. Corticothalamic resonance, states of vigilance and mentation. Neuroscience 2000;101:243-76. 
Thakor NV, Webster JG. Ground-free ECG recording with two electrodes. IEEE Trans Biomed Eng 1980;27:699-704.

Vergnes M, Marescaux C, Depaulis A, Micheletti G, Warter JM. Spontaneous spike and wave discharges in thalamus and cortex in a rat model of genetic petit mal-like seizures. Exp Neurol 1987;96:127-36.
Winter RB, Webster JG. Reduction of interference due to common mode voltage in biopotential amplifiers. IEEE Trans Biomed Eng 1983;30:58-62.

Wood DE, Ewins DJ, Balachandran W. Comparative analysis of power-line interference between two- or three-electrode biopotential amplifiers. Med Biol Eng Comput 1995;33:63-8. 